\title{
The Trapezoidal Dielectric Resonator Antenna
}

\author{
Georgios Almpanis, Student Member, IEEE, Christophe Fumeaux, Member, IEEE, and \\ Ruediger Vahldieck, Fellow, IEEE
}

\begin{abstract}
The operational concepts of the trapezoidal dielectric resonator antennas (DRAs) are presented and their performance is studied theoretically and experimentally. The rectangular parallelepiped is a special case of the more general trapezoid and therefore a comparison between both is useful in order to highlight their similarities and differences. It is shown in this paper that the inverted trapezoidal DRA exhibits a significantly larger impedance bandwidth than the rectangular parallelepiped, while the rest of their properties remain similar. Based on that, two versions of a probe-fed wide-band linearly polarized (LP) DRA are designed by combing the resonances of the first two lower-order modes of the trapezoidal DRA. The resulting impedance bandwidth exceeds $55 \%\left(S_{11}<-10 \mathrm{~dB}\right)$ and the patterns are broadside with low cross-polarization. The antennas are simple to fabricate and they can be easily made to be mechanically stable. The numerical analysis was performed with a commercially available FEM solver and an in-house developed finite-volume-time-domain (FVTD) code. The measurements confirmed the results of the simulations.
\end{abstract}

Index Terms-Dielectric resonator antennas (DRAs), finite-volume time-domain (FVTD), inverted trapezoidal shape, linear polarization, probe-feeding.

\section{INTRODUCTION}

D IELECTRIC resonator antennas (DRAs) were first introduced by Long et al. [1] in 1983. Before that, the high unloaded Q-factors of the dielectric resonators (DRs) made them very useful for various filter applications in microwave circuits. It was observed, however, that DRs placed in open environments had lower-order modes that exhibited low radiation Q-factors and therefore high "radiation losses." This led to the first dielectric structures being used as radiating elements. Since then, DRAs have received increasing attention due to their inherent attributes of low dissipation loss, high radiation efficiency and low mutual coupling in array configurations. Moreover, their high permittivity contributes to a significant reduction in their size, making them compact and light. The DRA versatility in shape and feeding mechanism gives the antenna designers the freedom to choose between many different options in order to obtain desired radiation characteristics such as linear or circular polarization and broadside or monopole-like radiation patterns. Finally, compared to the microstrip antennas, DRAs exhibit lower radiation Q-factors and thus wider impedance bandwidth.

Manuscript received November 9, 2007; revised March 5, 2008. Published September 4, 2008 (projected). This work was supported by ETH Research Grant TH-38/04-1.

The authors are with the Laboratory for Electromagnetic Field Theory and Microwave Electronics (IFH), Swiss Federal Institute of Technology (ETH), Zurich 8092, Switzerland (e-mail: almpanis@ifh.ee.ethz.ch).

Color versions of one or more of the figures in this paper are available online at http://ieeexplore.ieee.org.

Digital Object Identifier 10.1109/TAP.2008.928787
The DRA geometry in combination with its feeding scheme defines the radiation characteristics of the antenna. A simple dielectric rectangular parallelepiped excited through a coaxial probe or a slot can exhibit broadside, linearly polarized radiation characteristics, provided it is excited in its fundamental $\mathrm{TE}_{111}$ mode [2]. Similar characteristics are obtained if the fundamental $\mathrm{HEM}_{11 \delta}$ mode of the cylindrical DRA is excited [3], while some higher order modes exhibit patterns with a null in the broadside direction $\left(\theta=0^{\circ}\right)$. Circular polarization is also very easily attainable, provided the DRA shape and the coupling scheme lead to the excitation of two degenerate orthogonal modes with $90^{\circ}$ phase difference between them [4], [5].

Besides optimizing radiation characteristics and polarization purity, DRA designers are also faced with widening the bandwidth. Modern communications set the bandwidth requirements very high and DRA technology needs to keep up with them. Recent efforts towards this objective demonstrate impedance bandwidths ranging between $30 \%$ and $60 \%$ [6]-[8] for broadside patterns. In [6], an aperture coupled flipped staired pyramidal DRA exhibits an impedance bandwidth of $62 \%$ and broadside radiation patterns. In [7], the lower-order $\mathrm{TE}_{111}$ and $\mathrm{TE}_{113}$ modes of the rectangular DRA (RDRA) are excited at nearby frequencies through a strip while in [8], the fundamental $\mathrm{HEM}_{11 \delta}$ mode of the cylindrical DRA and the slot modes of the double-bowtie feeding scheme are combined in order to achieve wider impedance bandwidth. In those latter DRA geometries, the radiation patterns remain broadside and the polarization is of high purity for the entire frequency range of interest due to the horizontal-magnetic-dipole-like radiation of the resonant modes. Finally, a hybrid antenna consisting of an annular DRA combined with a quarter-wave monopole demonstrates $100 \%$ bandwidth and monopole-like patterns [9].

In this work, the properties of the probe-fed trapezoidal dielectric resonator antenna are investigated. The dielectric trapezoid can be treated as a geometrical extension of the dielectric rectangular parallelepiped and therefore the lowest-order mode of the trapezoid can be regarded as a pseudo $\mathrm{TE}_{111}$ mode. This pseudo mode exhibits similar radiation characteristics but a different radiation Q-factor as the $\mathrm{TE}_{111}$ mode of the RDRA. In the case of the inverted trapezoidal DRA (Fig. 1), the radiation Q-factor of the lowest-order mode is lower and hence the impedance bandwidth wider. This operational concept can be extended to higher order modes, so that a very wide operational bandwidth can be obtained. The resulting antenna consists of a rigid, easy to fabricate dielectric resonator using the very simple coaxial probe as a feeding scheme. A prototype has been fabricated and measured, to validate the results of the simulation.

The antenna proposed here is based on an operational concept similar to that of the flipped staired pyramidal DRA presented in [6]. However, the present device appears simple to design and 


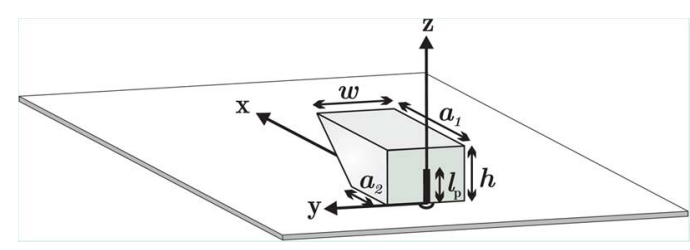

Fig. 1. Probe-fed trapezoidal DRA.

convenient to realize. The antenna shown in [6] involves producing three different rectangular slabs, aligning them so that they will be centered and ensuring no air gap between them. In addition, the design of such an antenna at another frequency band might encounter some difficulties, because the type and the properties of the modes excited are not clearly identified. In the DRA proposed here, the dielectric resonator shape is a simple trapezoid with constant width and height and the excited modes are very similar to the RDRA modes, as it will be shown in Section II.

The paper is organized as follows. In Section II, the trapezoidal DRA is compared to the dielectric rectangular parallelepiped in their lowest-order mode. The similarities and differences of the two geometries are discussed, while the advantages of the trapezoid over the rectangle are highlighted. Section III demonstrates the design of a very wideband linearly polarized (LP) trapezoidal DRA, whose wide bandwidth is the result of the excitation of pseudo $\mathrm{TE}_{111}$ and $\mathrm{TE}_{113}$ modes at slightly offset frequencies. Finally, Section IV summarizes the results of the investigations conducted here.

\section{The Dielectric Parallelepiped Versus The TRAPEZOID}

The probe-fed RDRA has been the subject of extensive investigations in the past ([2] and [7]). If operated in its fundamental $\mathrm{TE}_{111}$ mode, a DRA exhibits an impedance bandwidth of about $10 \%-15 \%$ (for a permittivity of about 10 ), high efficiency, low cross-polarization and broadside radiation patterns. The resonant frequency of the $\mathrm{TE}_{111}$ mode can be easily determined through the waveguide model method. The corresponding transcendental equation can be found in theory [10].

A careful consideration of the trapezoidal DRA geometry (Fig. 1) leads to the observation that the depicted trapezoid is a generalization of the rectangular parallelepiped. In other words, provided that the height $h$ and the width $w$ of the trapezoid are constant, the trapezoid becomes a rectangular parallelepiped, if its lengths $a_{1}$ and $a_{2}$ are chosen to be equal.

To compare the operation of the rectangular and the trapezoidal DRAs when excited in their lowest order mode, these geometries were investigated numerically and experimentally. The numerical analysis was performed using the commercial full-wave analysis tool Ansoft HFSS employing the finite element method (FEM). Fig. 1 illustrates the geometry of the considered DRAs, which were probe-fed trapezoids of dielectric constant $\varepsilon_{r d}=10$, height $h=7.6 \mathrm{~mm}$ and width $w=6 \mathrm{~mm}$. The lengths $a_{1}$ and $a_{2}$ were varied according to the relations $a_{1}=a+\Delta x$ and $a_{2}=a-\Delta x$, where $a=9 \mathrm{~mm}$. In all
TABLE I

SimUlated RESONANT FREQUENCY AND FRACTIONAL BANDWIDTH OF TRAPEZOIDS WITH $a=9 \mathrm{~mm}, w=6 \mathrm{~mm}, h=7.6 \mathrm{~mm}$ AND $\varepsilon_{r d}=10$

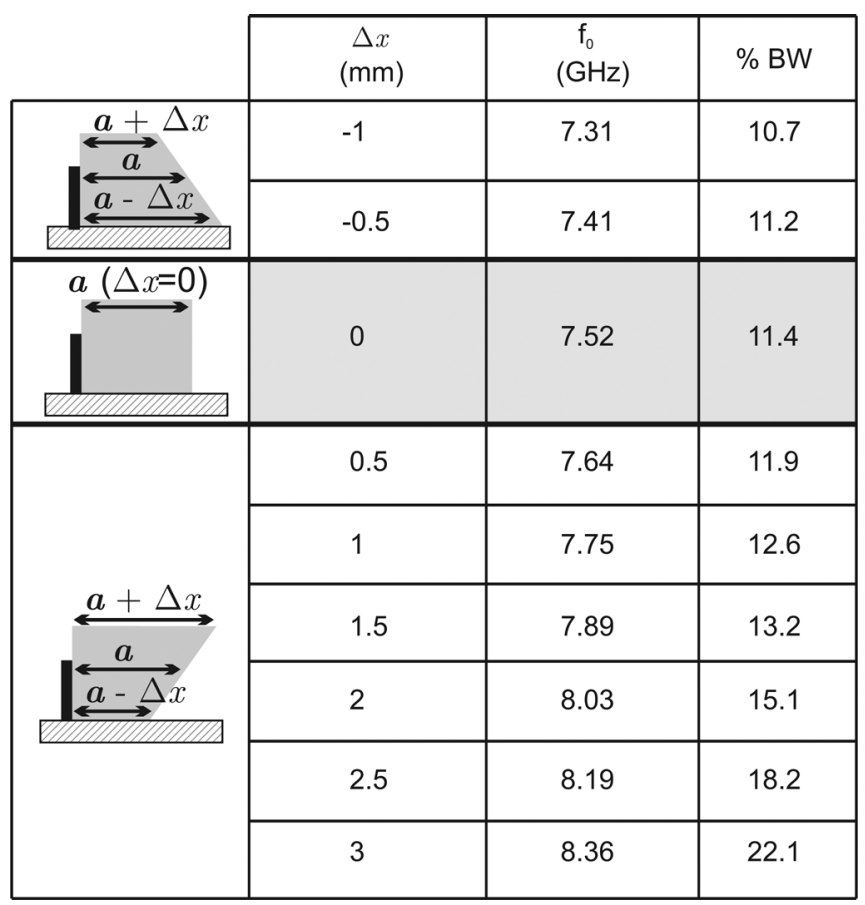

those geometries the total volume of the dielectric remains unchanged.

The results of the numerical analysis are summarized in Table I, where the DRA resonant frequency (zero-crossing of the input reactance) and fractional bandwidth $\left(S_{11}<-10 \mathrm{~dB}\right)$ are given for different values of $\Delta x$. It can be observed that an increase of $\Delta x$ from -1 to 3 (in other words, a geometrical change from the regular trapezoid to the rectangular parallelepiped and then to the inverted trapezoid) results in a monotonous increase of the resonant frequency and of the fractional bandwidth for the lowest order $\mathrm{TE}_{111}$ mode. Therefore, the inverted trapezoidal geometry $(\Delta x>0)$ exhibits a significantly wider operation band compared to the rectangular parallelepiped $(\Delta x=0)$ and the regular trapezoid $(\Delta x<0)$. This can be explained through the observation that in the inverted trapezoids the field lines are not well confined inside the dielectric resonator and therefore the radiation $\mathrm{Q}$ factor is bound to be smaller.

The bandwidth enhancement achieved through the inverted trapezoids has certain limitations. First, for the same volume of the dielectric resonator, the inverted trapezoidal DRAs resonate at higher frequencies and thus are electrically larger. Second, the asymmetry of the trapezoidal DRA in the xz plane (Fig. 1), leads to less symmetrical radiation patterns in the E-plane. To demonstrate this, Fig. 2 illustrates the dependence of the radiation patterns (E-plane) asymmetry on $\Delta x$ at the frequency of the lowest order mode excitation. The dimensions $a, w$ and $h$ of the trapezoid are the same as before. The asymmetry of the radiation patterns in the E-plane is defined as the ratio (expressed in $\mathrm{dB}$ ) of the DRA's gain at the elevation angles $+\theta$ and $-\theta$ from broadside, with $\theta$ ranging from $10^{\circ}$ to $70^{\circ}$. It is worth 


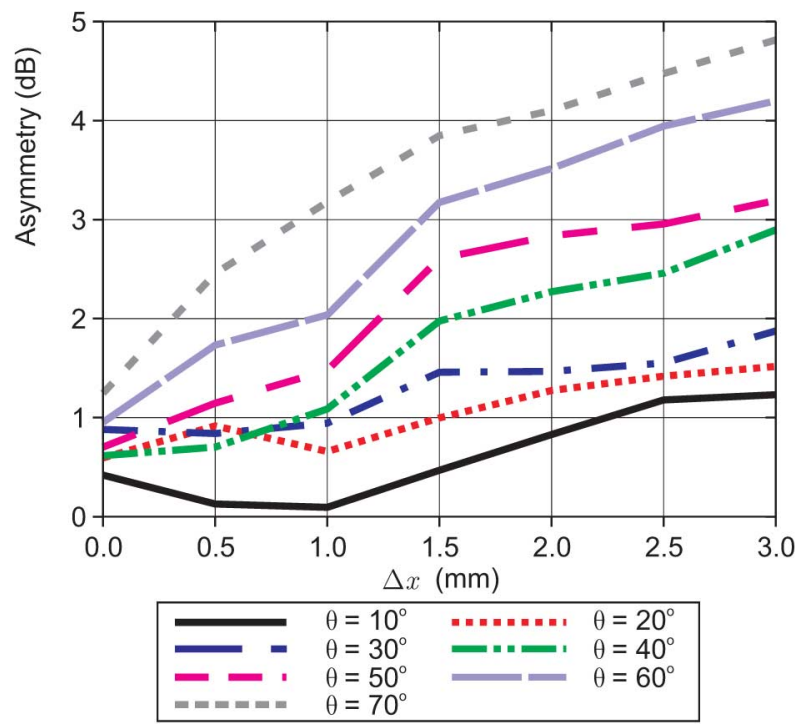

Fig. 2. Asymmetry in the E-plane versus $\Delta x$ at the frequency of the excitation of the lowest order mode.

mentioning here that the H-plane patterns are not affected by the trapezoidal shape, since the width of the trapezoid remains constant. Therefore, the patterns in the H-plane remain symmetrical.

As observed in Fig. 2, the asymmetry of the E-plane patterns increases with $\Delta x$. The more the DRA geometry deviates for the rectangular parallelepiped, the more asymmetrical the patterns become, especially at elevation angles above $60^{\circ}$. It needs to be underlined that even in the case of the RDRA $(\Delta x=0)$, a small asymmetry of approximately $0.5-1.5 \mathrm{~dB}$ is encountered, although perfectly symmetrical patterns in both the E- and $\mathrm{H}$ planes should be expected. This is caused by the asymmetry introduced by the probe feeding. A different feeding scheme, for instance a slot coupled by a microstrip, would yield more symmetrical patterns. In the present work, however, the probe was chosen mainly because of its simplicity.

It is now clear that the lengths $a_{1}$ and $a_{2}$ cannot be chosen arbitrarily in order to obtain a large impedance bandwidth, as the asymmetry in the radiation planes must also be taken into consideration. However, the tradeoff is not dramatic. Even a small deviation from a rectangular to an inverted trapezoidal shape can lead to a substantial increase in the impedance bandwidth, without significantly deteriorating the radiation characteristics. It should be emphasized that this is achieved while using a very common feeding scheme and a DRA geometry that is very simple and easy to manufacture.

To further illustrate the advantages of the inverted trapezoidal DRA, a trapezoid with dielectric constant $\varepsilon_{r d 2}=10$ and dimensions $a_{1}=a+\Delta x=9+2.5=11.5 \mathrm{~mm}, a_{2}=a \Delta x=$ $9-2.5=6.5 \mathrm{~mm}, h=7.6 \mathrm{~mm}, w=6 \mathrm{~mm}$ is chosen. According to Table I, this trapezoid is resonant at $8.19 \mathrm{GHz}$ in its lowest order mode. In order to obtain operation at $7.52 \mathrm{GHz}$, where the RDRA $(\Delta x=0)$ is also resonant, a number of different approaches can be used. The approach of increasing the dielectric constant of the DRA is not suitable, since the higher $\varepsilon_{r d}$ would result in a higher radiation $\mathrm{Q}$ factor and therefore in

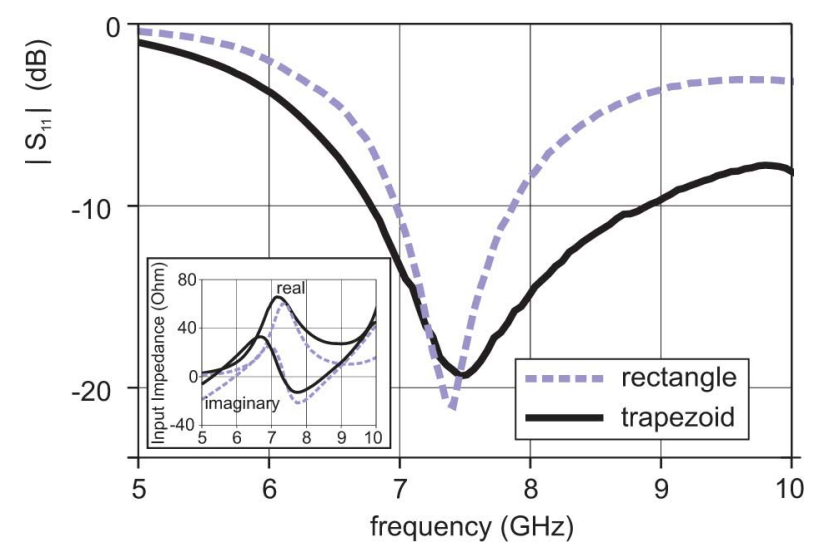

Fig. 3. Measured return loss and input impedance (inset) versus frequency for the rectangular and the trapezoidal DRAs.

a smaller bandwidth. The increase of just one of the dimensions of the DRA is also not considered due to the change of the ratios between the height, the width and the lengths of the trapezoid. Such an aspect ratio change is bound to alter the DRA operation, as it has already been shown for the canonical geometries [11]. The most suitable approach is to scale the trapezoid, so that it resonates at the desired frequency while the rest of the antenna characteristics remain unaltered. The finalized trapezoidal DRA has a dielectric constant of $\varepsilon_{r d 2}=10$ and dimensions $a_{1}=12.7 \mathrm{~mm}, a_{2}=7.2 \mathrm{~mm}, h=8.4 \mathrm{~mm}$ and $w=6.6 \mathrm{~mm}$. A prototype was manufactured and compared to the RDRA with dimensions $a_{1}=9 \mathrm{~mm}, h=7.6 \mathrm{~mm}$ and $w=6 \mathrm{~mm}$, as specified in Table I.

Fig. 3 depicts the measured return loss and the measured input impedance of the two DRAs versus frequency. Both antennas are resonant at approximately $7.4 \mathrm{GHz}$ (defined through the zero-crossing of the input reactance), indicating a $1.6 \%$ error from the numerically expected resonant frequency of $7.52 \mathrm{GHz}$. The RDRA exhibits a measured impedance bandwidth of approximately $11.9 \%$, which is almost half the bandwidth of $22 \%$ obtained by the trapezoidal DRA (19\% was expected from the simulations). The bandwidth enhancement for the trapezoidal DRA does not come along with a significant change in the operation of the lowest order mode, compared to the RDRA. To demonstrate that, the measured broadside gain versus frequency for both DRAs and their measured radiation patterns at $7.4 \mathrm{GHz}$ (E- and H- planes) are illustrated in Figs. 4 and 5, respectively. It can be observed that the measured broadside gain of the two DRAs differs by less than $0.3 \mathrm{~dB}$ in the frequency range between 7 and $8.5 \mathrm{GHz}$. In addition, their patterns at the frequency of excitation of their lowest-order mode $(7.4 \mathrm{GHz})$ show an identical horizontal-magnetic-dipole-like operation. The sole difference is that the E-plane patterns of the trapezoidal DRA exhibit an asymmetry, which however does not exceed 2-3 dB, even at large elevation angles.

Based on the above considerations, it is therefore clear that the lowest-order mode of the trapezoidal DRA has very similar characteristics with the $\mathrm{TE}_{111}$ mode of the RDRA. However, since the lowest-order trapezoidal mode does not abide by the transcendental equation for the $\mathrm{TE}_{111}$ mode [10], it will be called pseudo- $\mathrm{TE}_{111}$ mode. 


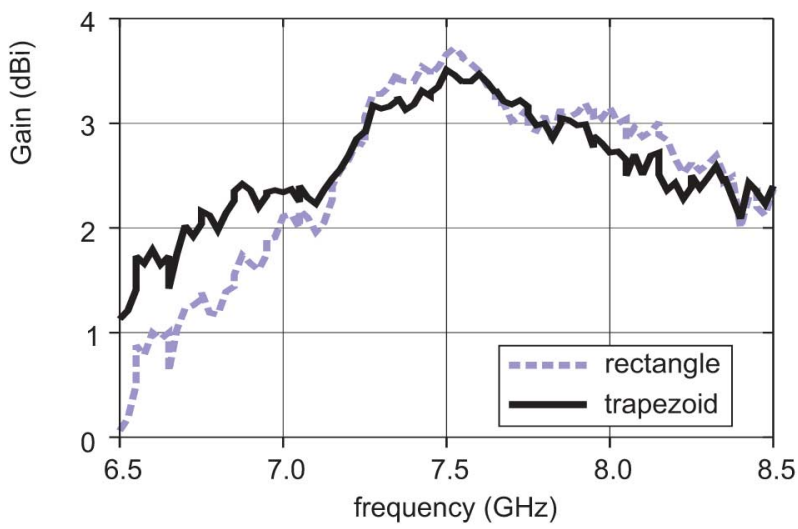

Fig. 4. Measured gain versus frequency for the rectangular and the trapezoidal DRAs.

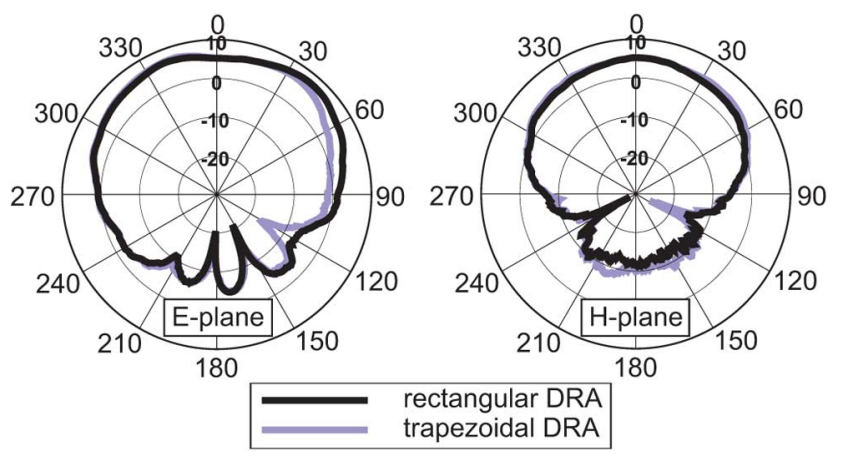

Fig. 5. Measured Radiation patterns of the rectangular and the trapezoidal DRAs in the E- and H-planes at 7.4 GHz.

To illustrate the generality of using an inverted trapezoid instead of a rectangular parallelepiped for bandwidth enhancement, a numerical investigation is carried out for DRAs of various dielectric constants. The objective is to make the DRA electrically smaller by increasing its dielectric permittivity $\varepsilon_{r d}$. Since this will result in the decrease of the DRA's impedance bandwidth [10], it is important to look into the impedance bandwidth achieved through the inverted trapezoidal shape compared to the parallelepiped. The results of this investigation are summarized in Fig. 6, where the fractional bandwidth of the rectangular and the trapezoidal DRAs is plotted versus their dielectric constant for a fixed operation at 7.4 GHz. The dimensions of the rectangular and the trapezoidal DRAs are $a_{1 \_ \text {rec }}=s \cdot 9 \mathrm{~mm}$, $h_{\text {rec }}=s \cdot 7.6 \mathrm{~mm}, w_{-r e c}=s \cdot 6 \mathrm{~mm}$ and $a_{1 \_ \text {trap }}=s \cdot 12.7 \mathrm{~mm}$, $a_{2 \text { trap }}=s \cdot 7.2 \mathrm{~mm}, h_{\text {trap }}=s \cdot 8.4 \mathrm{~mm}, w_{\text {trap }}=s \cdot 6.6 \mathrm{~mm}$ respectively. The constant $s$ is a scaling factor, denoting the decrease in the DRA dimensions when the dielectric constant is increasing beyond the reference value of $\varepsilon_{r d}=10$. Fig. 6 also includes the plot of the relative trapezoidal DRA volume compared to the volume of the original RDRA. This relative volume is plotted as a function of the dielectric constant for an operation at $7.4 \mathrm{GHz}$.

It is observed in the graph that the increase of the DRA dielectric permittivity leads, as expected, to the reduction of the fractional bandwidth. However, for all values of dielectric constant, the bandwidth obtained for the inverted trapezoid is at least 1.5 times larger than for the rectangular parallelepiped.

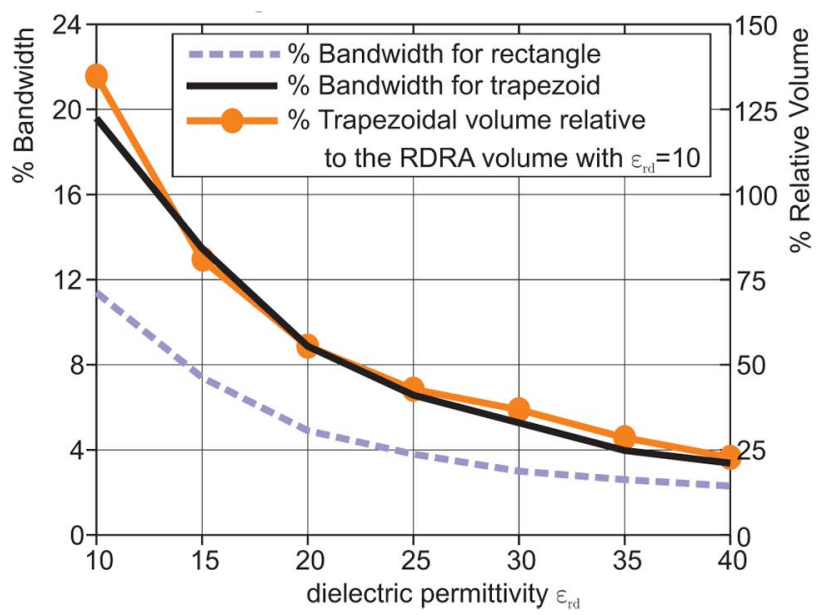

Fig. 6. Simulated fractional bandwidth and relative size of the rectangular and the trapezoidal DRAs versus their dielectric permittivity for a fixed operation at $7.4 \mathrm{GHz}$.

As an example, the RDRA of permittivity $\varepsilon_{r d}=10$ and dimensions $a_{1}=9 \mathrm{~mm}, h_{1}=7.6 \mathrm{~mm}, w_{1}=6 \mathrm{~mm} \mathrm{ex}-$ hibits an impedance bandwidth of $11.4 \%$, whereas the trapezoid of the same permittivity and dimensions $a_{2}=12.7 \mathrm{~mm}$, $a_{3}=7.2 \mathrm{~mm}, h_{2}=8.4 \mathrm{~mm}, w_{2}=6.6 \mathrm{~mm}$ (1.33 times bigger in volume than the parallelepiped) is by almost $70 \%$ more wideband at the same frequency of operation. It is interesting to observe here that the same bandwidth as of the RDRA can be obtained with a trapezoidal DRA of permittivity $\varepsilon_{r d}=17$ and with just $72 \%$ of the RDRA volume. It is thus obvious that for a certain frequency of operation and a predetermined required impedance bandwidth, size reduction can be achieved through the trapezoidal shape.

\section{WIDE-BAND TRAPEZOIDAL DRA}

The use of an inverted trapezoidal DRA has been demonstrated to be very advantageous compared to a RDRA on the criterion of the impedance bandwidth. In fact, with a suitable choice of the lengths $a_{1}$ and $a_{2}$ (Fig. 1) of the trapezoid, a bandwidth reaching or even exceeding $30 \%$ can be obtained. This, however, also results in asymmetrical E-plane patterns, which further deteriorate as the length $a_{2}$ becomes much larger than $a_{3}$.

One way to achieve a very wide impedance bandwidth without sacrificing well-behaved radiation characteristics is to employ the design concept introduced in [7]. In this paper, a simple strip-fed RDRA achieves an impedance bandwidth of around $42 \%$ by the careful optimization of the dielectric parallelepiped. It is well known from theory that the $\mathrm{TE}_{111}^{\mathrm{y}}$ and the $\mathrm{TE}_{113}^{\mathrm{y}}$ modes of the rectangular parallelepiped have similar radiation characteristics. Therefore, by properly designing the RDRA it is possible to excite the aforementioned two modes at slightly offset frequencies and hence to obtain a large operational bandwidth for the DRA.

A similar technique can be implemented for the inverted trapezoidal DRA, so that both the pseudo $\mathrm{TE}_{111}^{\mathrm{y}}$ and the pseudo $\mathrm{TE}_{113}^{\mathrm{y}}$ modes will be resonant at nearby frequencies. This is possible by simply increasing the height of the trapezoid with respect to its other dimensions. Figs. 7 and 8 depict two different 


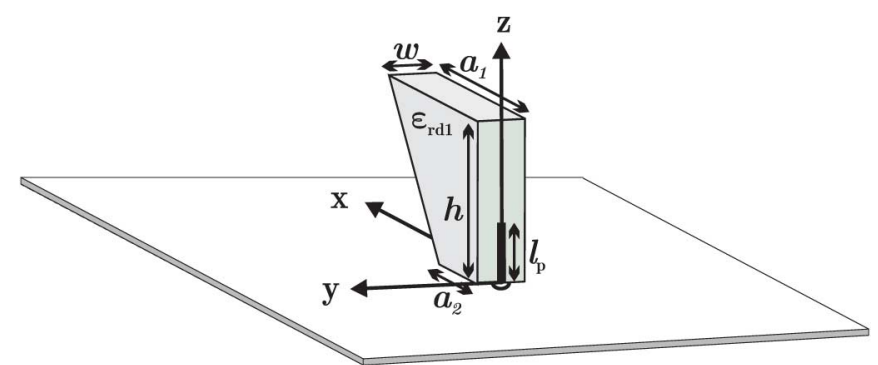

Fig. 7. High profile trapezoidal probe-fed DRA.

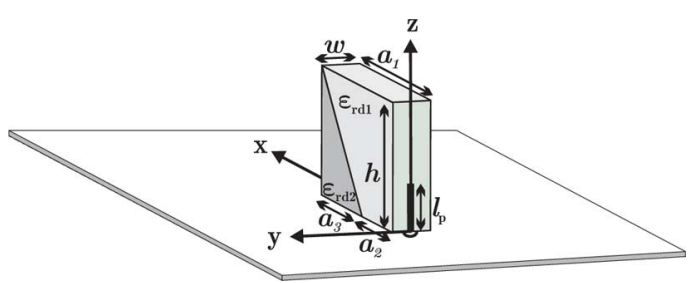

Fig. 8. Mechanically stable high profile inverted trapezoidal probe-fed DRA.

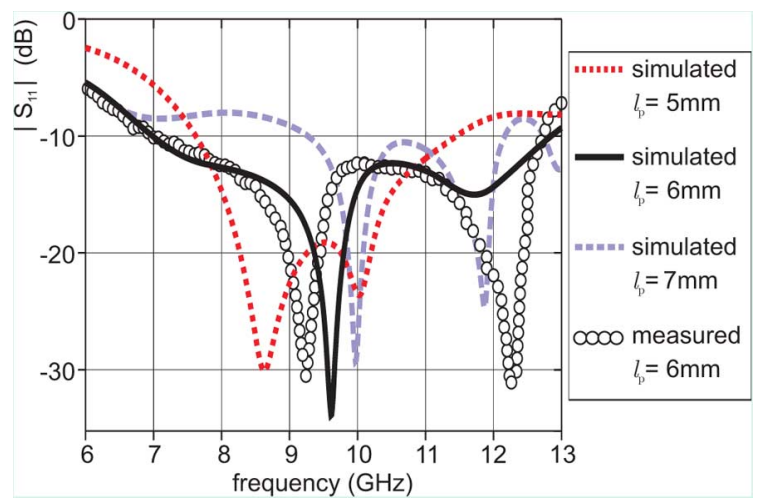

Fig. 9. Simulated (for different probe lengths) and measured (for best matching) return loss versus frequency of the trapezoidal DRA of Fig. 7.

implementations for wideband trapezoidal DRAs. In Fig. 7, a simple trapezoidal DRA is illustrated having a dielectric constant $\varepsilon_{r d 1}=10$ and dimensions $a_{1}=7 \mathrm{~mm}, a_{2}=10 \mathrm{~mm}$, $w=4 \mathrm{~mm}$ and $h=12 \mathrm{~mm}$. The DRA is fed through the center conductor of an SMA connector, which is protruding out of a small hole in a metallic plate of dimensions $140 \mathrm{~mm}$ $\times 120 \mathrm{~mm}$. The probe is situated in the middle of the narrow side of the dielectric resonator and it has a length $l_{\mathrm{p}}=6.0 \mathrm{~mm}$, optimized for best matching. The dependence of the matching on the probe length $l_{\mathrm{p}}$ is shown in Fig. 9, where the return loss of the trapezoidal DRA is plotted against frequency for various values of $l_{\mathrm{p}}$. For a probe length $l_{\mathrm{p}}=6.0 \mathrm{~mm}$ the -10 $\mathrm{dB}$ impedance bandwidth becomes approximately 59\%. The measured return loss in this case is in close agreement with the simulation.

A mechanically more stable version of the trapezoidal DRA is depicted in Fig. 8. The DRA consists of two parts; a trapezoid of dielectric constant $\varepsilon_{r d 1}=10$ and dimensions $a_{1}=7 \mathrm{~mm}$, $a_{2}=10 \mathrm{~mm}, w=4 \mathrm{~mm}$ and $h=12 \mathrm{~mm}$ and a plate of triangular cross-section with a dielectric constant $\varepsilon_{r d 2}=2.2$ and length $a_{3}=3 \mathrm{~mm}$, width $w=4 \mathrm{~mm}$ and height $h=12 \mathrm{~mm}$ that complements the rectangular shape. The feeding probe has
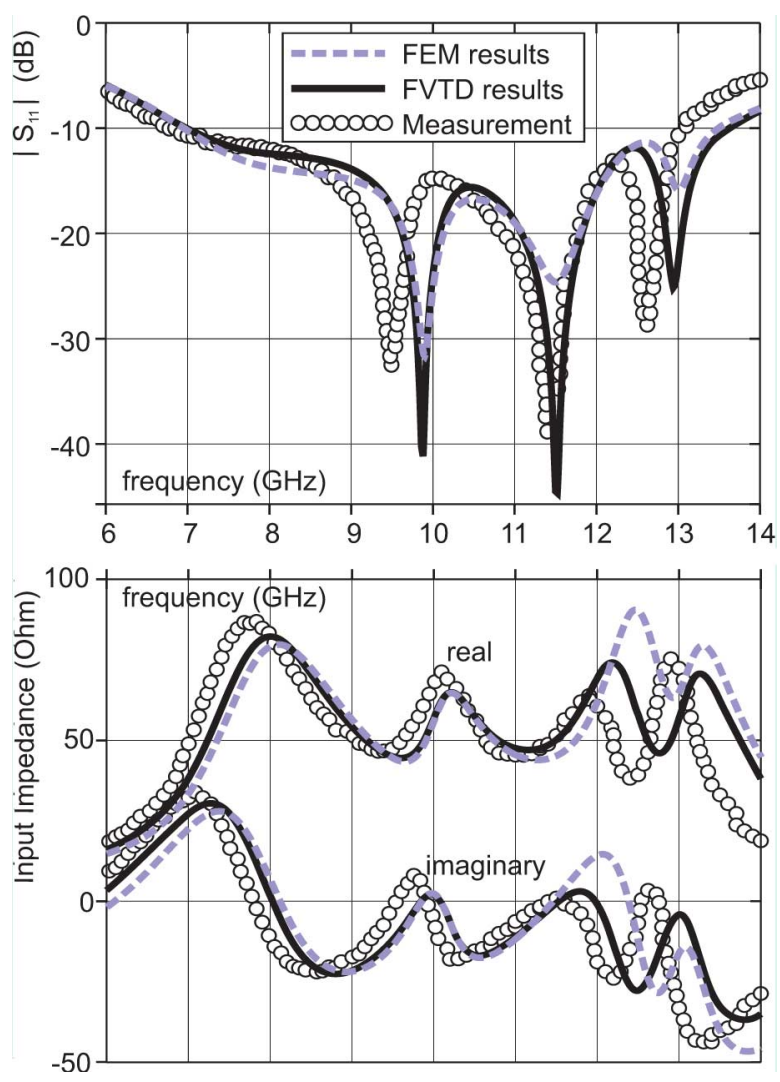

Fig. 10. Simulated (FEM and FVTD methods) and measured return loss and input impedance versus frequency for the trapezoidal DRA of Fig. 8.

a length $l_{\mathrm{p}}=6.2 \mathrm{~mm}$. The most important advantage of the new geometry is that the DRA is more rigid, since instead of a trapezoid, there is a rectangular parallelepiped now residing on the groundplane. This mechanical improvement does not come along with a cancellation of the wide bandwidth connected to the inverted trapezoidal geometry. The reason is that the dielectric constant of the triangular section is very low. This also results in the two DRAs of Figs. 7 and 8 operating in very similar ways. For the sake of brevity we will then concentrate on the DRA illustrated in Fig. 8.

A prototype of the mechanically stable trapezoidal DRA has been fabricated and measured. The plots of the return loss and the input impedance versus frequency are illustrated in Fig. 10, exhibiting an acceptable agreement between numerical and experimental results. The small discrepancy observed is most probably due to fabrication tolerances and the large standard deviation in the dielectric constant of the trapezoid $\left(\varepsilon_{r d 1} \approx 10 \pm 0.2\right)$. Fig. 10 displays, in addition to HFSS simulations, a numerical analysis performed with an in-house developed finite-volume time-domain (FVTD) solver [12]. The FVTD method combines an explicit time-stepping with a tetrahedral discretization. The agreement between the results from the two very different computational methods (time-domain versus frequency-domain, different in-cell approximations, different tetrahedral meshes) is striking and dissimilarities are only observed at high frequencies. This confirms that the small discrepancy between simulations and measured results can most likely be attributed to fabrication tolerances. 


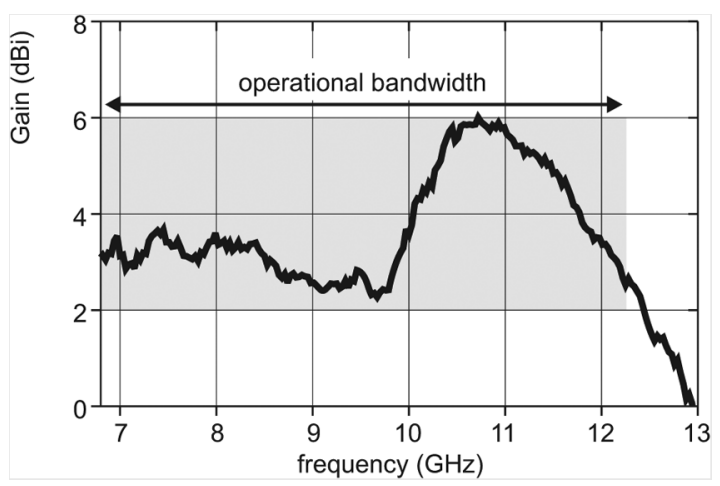

Fig. 11. Measured broadside gain versus frequency for the trapezoidal DRA.

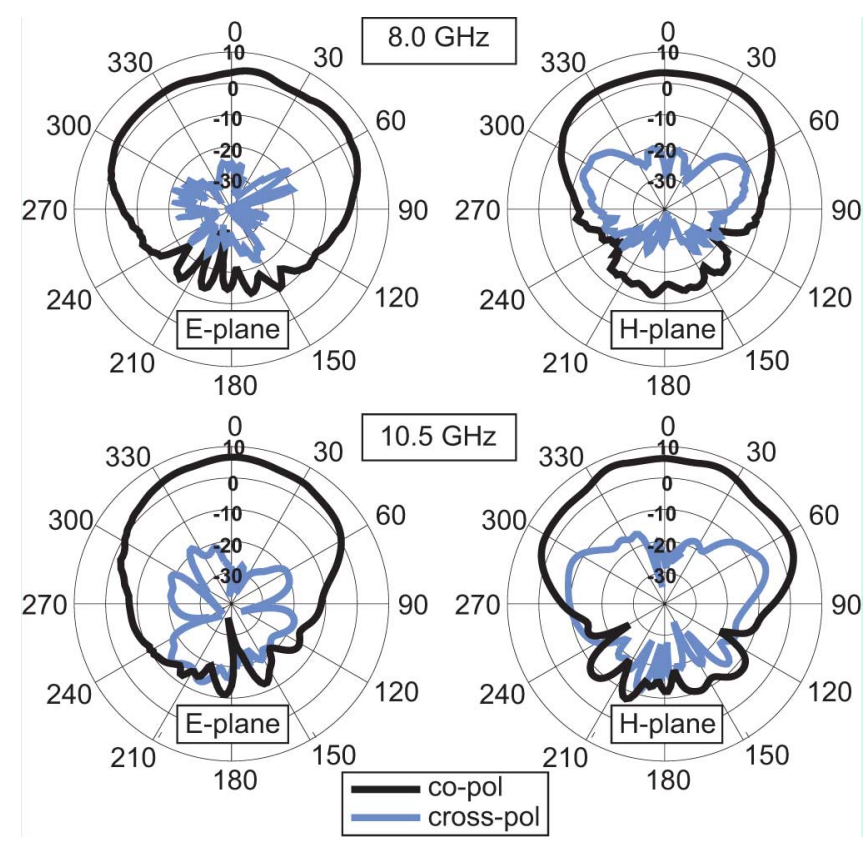

Fig. 12. Measured Radiation patterns in the E- and H-plane for the trapezoidal DRA ofFig. 8 at 8.0 and $10.5 \mathrm{GHz}$.

As observed from the input impedance curves, the trapezoidal DRA owes its $62 \%$ impedance bandwidth $(6.8-13 \mathrm{GHz})$ to the excitation of three modes: the $\mathrm{TE}_{111}^{\mathrm{y}}$, the $\mathrm{TE}_{113}^{\mathrm{y}}$ and a higher order mode. The first two modes are intentionally excited at nearby frequencies due to their similar radiation characteristics. To determine whether the operational bandwidth of the DRA can also include the third mode, the broadside gain of the DRA needs to be considered. Gain measurements are depicted in Fig. 11, giving a better insight to the DRA operation. In the frequency range between 6.8 and $9.5 \mathrm{GHz}$ the $\mathrm{TE}_{111}^{\mathrm{y}}$ mode is dominant, resulting in broadside operation with a gain of around $3 \mathrm{dBi}$. At $10 \mathrm{GHz}$ the $\mathrm{TE}_{113}^{\mathrm{y}}$ mode is excited; this leads to a broadside gain of 4-6 dBi. Finally, the excitation of the third mode at approximately $12 \mathrm{GHz}$ results in the rapid decrease of the broadside gain. Since well-behaved broadside DRA operation is desired, only operation up to around $12.2 \mathrm{GHz}$ is considered. Thus, the operational bandwidth of the DRA can be finally estimated to $57 \%$, with stable broadside patterns and good polarization purity.

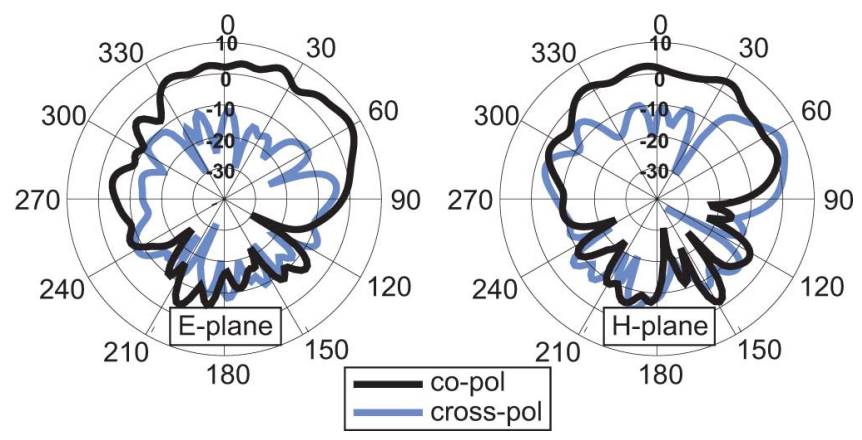

Fig. 13. Measured radiation patterns in the E- and H-plane for the trapezoidal DRA of Fig. 8 at $12.3 \mathrm{GHz}$.

The radiation patterns of the previously presented trapezoidal DRA are depicted in Fig. 12 at frequencies 8.0 and $10.5 \mathrm{GHz}$. The radiation is broadside within the entire operational bandwidth and the cross-polarization remains below $-15 \mathrm{~dB}$ for a wide range of elevation angles $\theta$. The minor ripples at around $\theta=0^{\circ}$ in the E-plane are the result of diffraction effects due to the finite size of the groundplane. Finally, the H-plane patterns are, as expected, perfectly symmetrical for all frequencies, whereas the E-plane patterns become less symmetrical with increasing frequency. This asymmetry can be kept, however, within acceptable limits when considering the design procedure described in Section II.

To further account for the gain drop above $12.2 \mathrm{GHz}$, the Eand $\mathrm{H}$-plane patterns of the trapezoidal DRA are illustrated in Fig. 13 at frequency $12.3 \mathrm{GHz}$. It can be observed that the patterns of this mode are characterized by an increased asymmetry in the E-plane due to the trapezoidal shape, higher levels of cross-polarization and some ripples at around $\theta \approx-60^{\circ}$. The broadside gain of this third mode is lower than of the $\mathrm{TE}_{113}^{\mathrm{y}}$ mode and it decreases further as the frequency increases. This is most probably due to the further deterioration of the E-plane patterns symmetry and the increase of the cross-polarization at higher frequencies.

\section{CONCLUSION}

A probe-fed trapezoidal dielectric resonator antenna has been investigated theoretically and experimentally. The advantages of such a geometry compared to a RDRA were highlighted, and the tradeoff between bandwidth and asymmetry of the radiation patterns was discussed. Finally, two versions of a wideband trapezoidal DRA were proposed, exhibiting a simple but insightful design, impedance bandwidth exceeding $55 \%$ and very well behaved radiation patterns.

\section{ACKNOWLEDGMENT}

The authors would like to acknowledge the valuable support from H.-R. Benedickter. The authors are also grateful to $\mathrm{M}$. Lanz for fabricating the feeding structure.

\section{REFERENCES}

[1] S. A. Long, M. W. McAllister, and L. C. Shen, "The resonant cylindrical dielectric cavity antenna," IEEE Trans. Antennas Propag., vol. 31, no. 3, pp. 406-412, May 1983. 
[2] R. K. Mongia and A. Ittipiboon, "Theoretical and experimental investigations on rectangular dielectric resonator antennas," IEEE Trans. Antennas Propag., vol. 45, no. 9, pp. 1348-1356, Sep. 1997.

[3] A. A. Kishk, A. Ittipiboon, Y. M. M. Antar, and M. Cuhaci, "Slot excitation of the dielectric disk radiator," IEEE Trans. Antennas Propag., vol. 43, no. 2, pp. 198-201, Feb. 1995.

[4] G. Almpanis, C. Fumeaux, and R. Vahldieck, "Offset cross-slot-coupled dielectric resonator antenna for circular polarization," IEEE Microw. Wireless Compon. Lett., vol. 16, pp. 461-463, Aug. 2006.

[5] H. K. Ng and K. W. Leung, "Frequency tuning of the linearly and circularly polarized dielectric resonator antennas using multiple parasitic strips," IEEE Trans. Antennas Propag., vol. 54, no. 1, pp. 225-230, Jan. 2006.

[6] R. Chair, A. A. Kishk, K. F. Lee, and C. E. Smith, "Wideband flipped staired pyramid dielectric resonator antennas," Electron. Lett., vol. 40, no. 10, pp. 581-582, May 2004.

[7] B. Li and K. W. Leung, "Strip-Fed rectangular dielectric resonator antennas with/without a parasitic patch," IEEE Trans. Antennas Propag., vol. 53, no. 7, pp. 2200-2207, Jul. 2005.

[8] G. Almpanis, C. Fumeaux, and R. Vahldieck, "Novel broadBand dielectric resonator antennas fed through double-bowtie-slot excitation scheme," ACES J., vol. 22, no. 1, pp. 97-104, Mar. 2007.

[9] M. Lapierre, Y. M. M. Antar, A. Ittipiboon, and A. Petosa, "Ultra wideband monopole/dielectric resonator antenna," IEEE Microw. Wireless Compone. Lett., vol. 15, pp. 7-9, Jan. 2005.

[10] D. Kajfez and P. Guillon, Dielectric Resonators. Norwood, MA: Artech House, 1986.

[11] K. M. Luk and K. W. Leung, Dielectric Resonator Antennas. Hertfordshire, U.K.: Research Studies Press, 2003.

[12] C. Fumeaux, D. Baumann, and R. Vahldieck, "Finite-Volume time-domain analysis of a cavity-backed archimedean spiral antenna," IEEE Trans. Antennas Propag., vol. 54, no. 3, pp. 844-851, Mar. 2006.

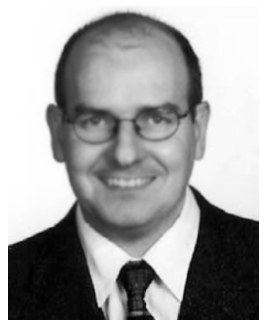

Christophe Fumeaux (M'03) received the Diploma and Ph.D. degrees in physics from the Swiss Federal Institute of Technology (ETH), Zurich, Switzerland, in 1992 and 1997, respectively.

From 1998 to 2000, he was a Postdoctoral Researcher involved in infrared technology with the School of Optics, University of Central Florida, Orlando. In 2000, he joined the Swiss Federal Office of Metrology, Bern, Switzerland, as a Scientific Staff Member. Since 2001, he has been a Research Associate with the Laboratory for Electromagnetic Fields and Microwave Electronics (IFH), ETH, Zurich, Switzerland. During the Fall of 2005, he was a Visiting Scientist with the Laboratory of Sciences and Materials for Electronics, and of Automatic (LASMEA), University Blaise Pascal, Clermont-Ferrand, France. His current main research interest concerns computational electromagnetics in the time domain for numerical analysis of microwave circuits and antennas.

Dr. Fumeaux has been the chairman of the IEEE Swiss Joint Chapter on Microwave Theory and Techniques, Antennas and Propagation, and EMC since January 2006. He was the recipient of the ETH Silver Medal of Excellence for his doctoral dissertation. He was the recipient of the Outstanding Paper Award of the Applied Computational Electromagnetics Society (ACES) in 2004.

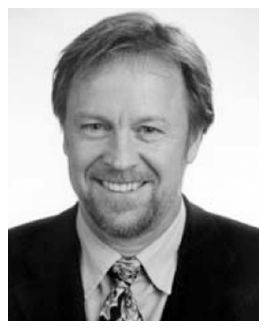

Ruediger Vahldieck (M'85-SM'86-F'99) received the Dipl.-Ing. and the Dr.-Ing. degrees in electrical engineering from the University of Bremen, Germany, in 1980 and 1983, respectively.

He was a Postdoctoral Fellow with the University of Ottawa, Ottawa, ON, Canada, until 1986. In 1986, he joined the Department of Electrical and Computer Engineering, University of Victoria, BC, Canada, where he became a Full Professor in 1991. During Fall and Spring of 1992 to 1993 he was a Visiting Scientist at the "Ferdinand-Braun-Institute fur Hochfrequenztechnik," Berlin, Germany. In 1997, he was appointed Professor for electromagnetic field theory at the Swiss Federal Institute of Technology, Zurich, and became head of the Laboratory for Electromagnetic Fields and Microwave Electronics (IFH) in 2003. In 2005, he became President of the Research Foundation for Mobile Communications and was elected Head of the Department of Information Technology and Electrical Engineering (D-ITET), ETH Zurich. Since 1981 he has published more than 300 technical papers in books, journals and conferences. His research interests include computational electromagnetics in the general area of EMC and in particular for computer-aided design of microwave, millimeter wave and optoelectronic integrated circuits.

Dr. Vahldieck received the Outstanding Publication Award of the Institution of Electronic and Radio Engineers in 1983, the K. J. Mitra Award of the IETE (in 1996) for the Best Research Paper in 1995, and the ACES Outstanding Paper Award in 2004. He is the Past-President of the IEEE 2000 International Zurich Seminar on BroadBand Communications (IZS'2000) and since 2003 President and General Chairman of the international Zurich Symposium on Electromagnetic Compatibility (EMC Zurich). He is a member of the editorial board of the IEEE Transactions on Microwave Theory and Techniques. From 2000 until 2003, he served as Associate Editor for the IEEE MICROWAVE AND WIRELESS COMPONENTS LETTERS and from July 2003 until the end of 2005 as the Editor-in Chief. Since 1992, he has been on the Technical Program Committee of the IEEE International Microwave Symposium, the MTT-S Technical Committee on Microwave Field Theory, and in 1999 on the TPC of the European Microwave Conference. From 1998 until 2003, he was the Chapter Chairman of the IEEE Swiss Joint Chapter on MTT, AP, and EMC. 